This item was submitted to Loughborough's Research Repository by the author.

Items in Figshare are protected by copyright, with all rights reserved, unless otherwise indicated.

\title{
Compressing computer network measurements using embedded zerotree
} wavelets

\author{
PLEASE CITE THE PUBLISHED VERSION \\ PUBLISHER \\ (c) IEEE
}

VERSION

VoR (Version of Record)

LICENCE

CC BY-NC-ND 4.0

\section{REPOSITORY RECORD}

Kyriakopoulos, Konstantinos G., and David J. Parish. 2019. "Compressing Computer Network Measurements Using Embedded Zerotree Wavelets”. figshare. https://hdl.handle.net/2134/4764. 
This item was submitted to Loughborough's Institutional Repository (https://dspace.lboro.ac.uk/) by the author and is made available under the following Creative Commons Licence conditions.

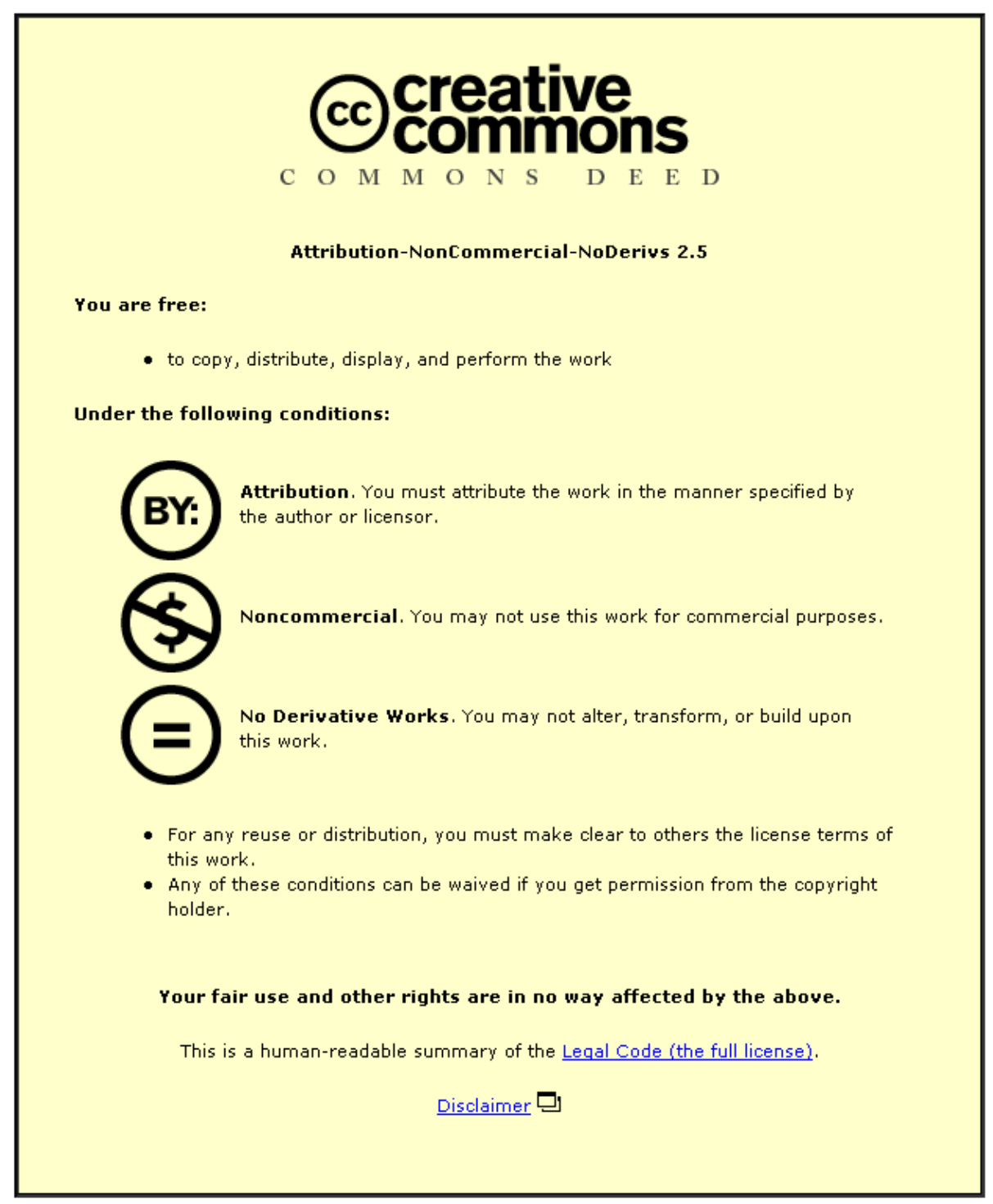

For the full text of this licence, please go to: http://creativecommons.org/licenses/by-nc-nd/2.5/ 


\title{
Compressing Computer Network Measurements Using Embedded Zerotree Wavelets
}

\author{
Konstantinos G. Kyriakopoulos, David J. Parish \\ Department of Electronic and Electrical Engineering \\ Loughborough University \\ Loughborough, LE11 3TU, U.K. \\ e-mail: \{elkk, d.j.parish\}@lboro.ac.uk.
}

\begin{abstract}
Monitoring and measuring various metrics of high data rate and high capacity networks produces a vast amount of information over a long period of time. Characteristics such as throughput and delay are derived from packet level information and can be represented as time series signals. This paper looks at the Embedded Zero Tree algorithm, proposed by Shapiro, in order to compress computer network delay and throughput measurements while preserving the quality of interesting features and controlling the level of quality of the compressed signal. The quality characteristics that are examined are the preservation of the mean square error (MSE), the standard deviation, the general visual quality (the PSNR) and the scaling behavior. Experimental results are obtained to evaluate the behaviour of the algorithm on delay and data rate signals. Finally, a comparison of compression performance is presented against the lossless tool bzip2.
\end{abstract}

\section{Introduction}

Monitoring and measuring various metrics of high speed and high capacity networks produces a vast amount of information over a long period of time. These metrics describe the status and performance of the network in terms of utilization, congestion, packets lost, etc, and provide important information regarding the operation of the network over time. The storage of the produced measurements is practically inefficient, as they require a very large volume of data. This itself significantly increases the cost for measuring high-speed networks. For this reason, there is a need to derive an efficient method of data analysis and reduction in order to archive and store the enormous amount of measurements describing the monitored traffic.

Satisfying this need is useful for researchers and network administrators particularly if they run experimental proto- cols on a monitored network. The researchers would like to know how their algorithms affect the network's characteristics in terms of utilization, delay, data rate, etc. Such characteristics are derived from packet level information and many can be represented as time series signals. Such signals can be analyzed and compressed using a signal analysis technique.

By using a threshold to choose a few wavelet domain coefficients, wavelets can represent an approximation of the analyzed signal in a compact form. This makes wavelets an appropriate choice for compression applications [3]. Wavelets have the ability to detect characteristics of nonstationary signals due to their finite nature that describes local features better than say sinusoids. Non-stationary signals are stochastic signals whose statistical properties change with time [1]. Such signals are met in many areas of research including computer network measurements.

To date, wavelets have been generally used to detect network performance problems. They have been applied to traffic rate signals in order to infer the time scale associated with the dominant round trip time (RTT) through the examination of the energy function of the detail coefficients [6]. They have also been used for de-noising one-way delay signals in order to detect shared congestion between different flows [8]. [5] shows that wavelet filters are quite effective at exposing the details and characteristics of ambient and anomalous traffic. [10] presents an off-line technique that is complimentary to sampling approaches for reduction of pre-captured data traffic packets while accurately preserving mean and standard deviation. This technique is useful for accurately modeling network traffic.

Previous work by the authors [9] has implemented wavelet coefficient thresholding for performing lossy compression while maintaining the quality of interesting features of the signals. The thresholding method applied in that work was proposed by Gupta and Kaur (GK), and gave a good balance between compression ratio (C.R.) and Peak 
Signal to Noise Ratio (PSNR) results.

The GK threshold selection calculated statistics from the absolute value of the non-zero coefficients and used these to select a threshold. However, in some cases, some important coefficients were "hidden" by their statistical insignificance and thus were discarded. This had a negative effect on the level of quality in the reconstructed form of some signals. An extension to this work would therefore be a method of controlling the quality of the decompressed signal.

In this paper Shapiro's proposed Embedded Zero-tree Wavelet (EZW) algorithm [11] is used in order to develop a method for compressing various computer network measurements while having control over the reconstructed signal's quality. Embedded encoding or progressive encoding is a method of compressing with increasing accuracy, i.e. the produced bits are generated in order of importance. For a specific bit rate encoding, all encodings of the same signal at lower bit rates are included in the beginning of the bit stream.

The rest of the paper is structured as follows. In section 2, the goals of our compression technique are described along with what characteristics need to be preserved and what they mean. In section 3 the methodology followed for producing the off-line results is presented and the results are given in section 4. Finally, conclusions and ideas for future work are given in section 5 .

\section{Goals of Compression}

The algorithm should maintain the characteristics and interesting features of the original examined signal. Experimental results were obtained to examine how the applied method behaves in terms of C.R. Furthermore, apart from high compression ratios and good reconstruction signal quality, the following factors of the compressed signal were investigated in order to determine the effect of compression on them: 1.High compression ratios, 2. Quality characteristics ( Preserve sudden changes: peaks and dips, High PSNR), 3. Statistical characteristics (Preserve Mean and standard deviation, Energy and Scaling Behavior).

\subsection{Spikes and overall quality}

When times of very high network usage occur, spikes in the data traces are reported. On a public network, such spikes would be of considerable importance as they indicate periods of changing load and their further investigation may indicate if changes to the network are required. On a research network such as JANET Lightpath [4], analysis of the spikes may indicate what impact a particular experiment has had on a given network path. Preservation of these spikes for later analysis is therefore of great importance.

\subsection{Energy and Scaling Behavior}

The energy function plot [2] was used in order to examine the preservation of the scaling behavior after compression. A statistic known as the energy function $E_{j}$ indicates the average energy of the arrival process contained at scale $j$ and is defined by [2]:

$$
E_{j}=\frac{1}{N_{j}} \sum_{k}\left|d_{j, k}\right|^{2}, \quad j=1,2, \ldots, n
$$

where $N_{j}$ is the number of wavelet ("detail") coefficients at scale $j$.

The energy plot can be generated by plotting $\log \left(E_{j}\right)$ against the scale $j$ from finer to coarser scales. Intuitively, this plot illustrates the scaling behaviour of the underlying time series (such as a traffic arrival process) at different time-scales.

\section{Methodology}

For the purpose of examining the behaviour of the EZW technique, 30 delay and 30 data rate measurement signals were used as inputs to the algorithm. The data rate signals were taken from a real commercial network and the delay signals were generated in a test bed. Each signal had 1024 measurement points and was decomposed using the Haar wavelet at level 9 because the EZW algorithm requires 2 approximation coefficients after the analysis.

The Haar wavelet is the simplest wavelet algorithm that can give perfect reconstruction and has the following advantages [9]: It is conceptually simple, fast, memory efficient and exactly reversible without producing edge effects. Because of the above advantages and the best reconstruction results observed from experiments [9], the Haar wavelet was chosen for the off-line experiments.

The methodology flow chart is presented in Fig. 1 and incorporates the EZW procedure described in [11]. After the wavelet decomposition, the EZW encoder attempts to encode the wavelet coefficients by sequentially dividing the threshold by two. In each run, after decoding the symbols, the inverse wavelet transform is applied and the PSNR of the reconstructed signal is calculated to estimate the reconstruction quality. However, high visual quality does not guarantee preservation of the energy scale and thus the energy of the reconstructed signal in all scales needs to be calculated.

If the PSNR value is not higher than a predetermined desired value ( $X$ in Fig. 1) and if the energy difference in any scale is higher than a desired threshold $(Y=1 d B$ in Fig. 1), then the EZW encoder continues to halve the threshold and encodes more detail coefficients. Otherwise, the outputs from the dominant and subordinate passes are passed through the arithmetic encoder. 


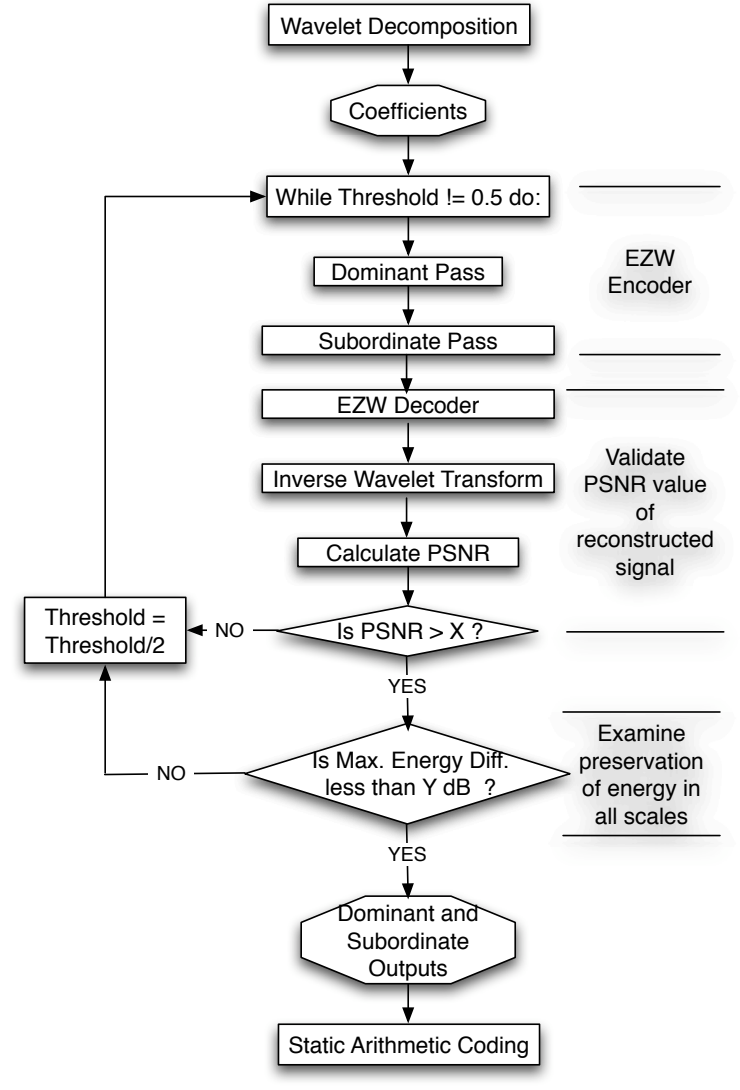

Figure 1. Methodology algorithm. $X$ is the desired target PSNR value

To reconstruct the signal, the stored arithmetic coded output is passed through the arithmetic decoder, the EZW decoder and lastly through the inverse wavelet algorithm.

The individual C.R. results are calculated from the ratio of the original file size over the sum of file sizes of the encoded dominant and sub-ordinate file sizes. The following PSNR and C.R. values in section 4 refer to the average over the 30 signals (delay or data rate) unless stated otherwise. In addition to the quantitative measurement of the reconstruction quality, we also include figures presenting the original signal, the reconstructed signal after compression and the error between them.

\section{Results}

Table 1 and Table 2 show the average, minimum and maximum PSNR and average C.R. results over 30 experiments for delay and data rate signals respectively. Empirically, for both delay and data rate signals, PSNR values more than $44 \mathrm{~dB}$ give very good visual results (signal indistinguishable from reconstruction), PSNR values less than
Table 1. Target, average, min. and max. PSNR values from 30 runs and C.R. values for delay signals.

\begin{tabular}{|c||c|c|c|c|}
\hline Target PSNR & Avg. & Min. & Max. & C.R. \\
\hline \hline $50 \mathrm{~dB}$ & 52.52 & 50.12 & 56.75 & 7.54 \\
\hline $48 \mathrm{~dB}$ & 50.97 & 48.28 & 54.81 & 8.47 \\
\hline $46 \mathrm{~dB}$ & 46.16 & 49.14 & 51.59 & 9.66 \\
\hline $44 \mathrm{~dB}$ & 46.82 & 44.06 & 50.73 & 11.4 \\
\hline $42 \mathrm{~dB}$ & 44.68 & 42.06 & 48.00 & 13.06 \\
\hline $40 \mathrm{~dB}$ & 42.15 & 40.06 & 46.16 & 16.09 \\
\hline $38 \mathrm{~dB}$ & 40.45 & 38.08 & 44.06 & 18.76 \\
\hline $36 \mathrm{~dB}$ & 38.23 & 36.50 & 41.44 & 22.7 \\
\hline
\end{tabular}

Table 2. Target, average, min. and max. PSNR values from 30 runs and C.R. values for data rate signals.

\begin{tabular}{|c||c|c|c|c|}
\hline Target PSNR & Avg. & Min. & Max. & C.R. \\
\hline \hline $44 \mathrm{~dB}$ & 48.39 & 44.03 & 51.03 & 11.16 \\
\hline $42 \mathrm{~dB}$ & 44.17 & 42.27 & 49.12 & 13.46 \\
\hline $40 \mathrm{~dB}$ & 43.70 & 41.74 & 46.87 & 13.76 \\
\hline $38 \mathrm{~dB}$ & 43.22 & 38.02 & 44.75 & 14.21 \\
\hline $36 \mathrm{~dB}$ & 37.69 & 36.11 & 43.29 & 20.69 \\
\hline
\end{tabular}

$35 \mathrm{~dB}$ loose some of the important signal characteristics while PSNR values less than $30 \mathrm{~dB}$ are not acceptable for such signals. The target PSNR is set from $36 \mathrm{~dB}$ up to a PSNR value at which the energy is preserved in all scales (as discussed in section 3). For the examined delay signals this is $50 \mathrm{~dB}$ but for data rate signals the energy can be preserved with much lower PSNR as will be explained later.

As expected the higher the target PSNR, the lower the achievable C.R. Data rate signals are compressed less than delay signals because they include more fluctuation, which amounts to high frequency components that need to be preserved in order for the quality to reach the required PSNR value.

By lowering the threshold more detail is encoded by the EZW algorithm in order to increase the quality of the reconstructed signal and reach a specified target PSNR value. That value, however, indicates a lower limit that the quality should have and the average PSNR is usually higher than that. This happens because by lowering the threshold, the PSNR increases by fractions of dBs up to several dBs, depending on the signal. This is why in Tables 1 and Table 2 
the average PSNR produced is higher than the target PSNR.

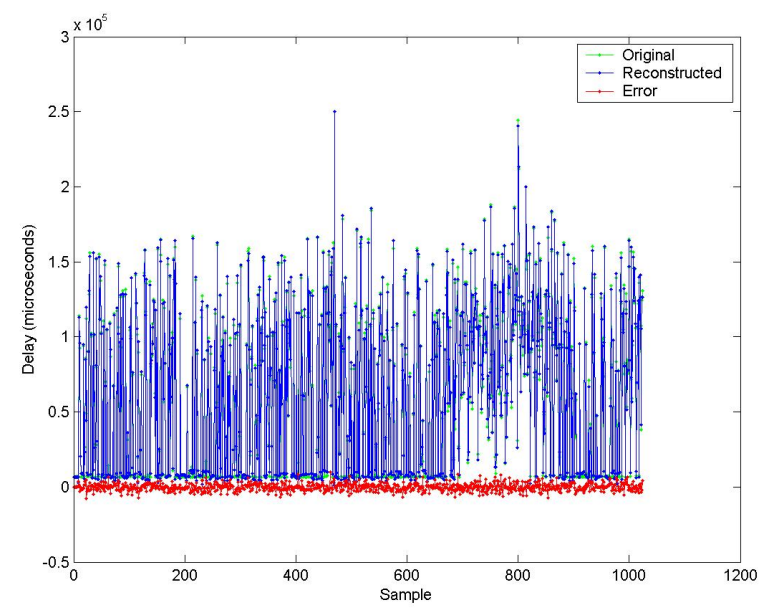

Figure 2. Delay signal 10 with $P S N R=40 \mathrm{~dB}$ and C.R. $=6.5$

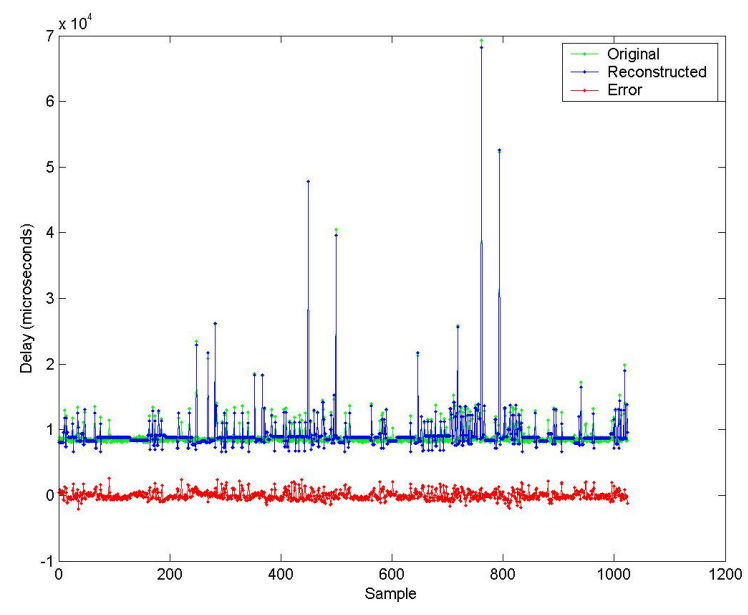

Figure 3. Delay signal 24 with PSNR=40.27 dB and C.R.=16.97

For a target PSNR of $40 \mathrm{~dB}$ Fig. 2 and 3 show two delay signals before and after the compression. The error is also given for easy judgement of the reconstruction quality. For delay signals the achieved average PSNR is $42.15 \mathrm{~dB}$. The overall compression is 16 times.

Signal 10 (Fig. 2) differed from the other delay signals used in the experiments because it is very bursty. The high burtsiness of the signal makes it difficult to compress as it requires a lot of high frequency components to be preserved. This is why in that case C.R. is 6.5.

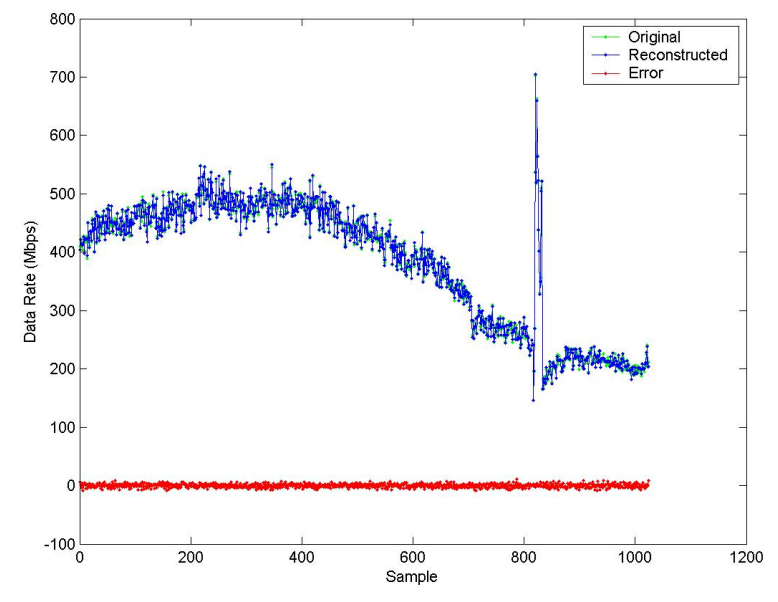

Figure 4. Data rate signal 20 with $P S N R=44.5$ $\mathrm{dB}$ and C.R.=13.67

Similarly for the same target quality Fig. 4 and 5 show two data rate signals. For data rate signals the achieved average PSNR is $43.70 \mathrm{~dB}$. The overall compression is more than 13 times.

Tables 3 and 4 show the average percentage relative error for standard deviation and mean for all 30 delay and data rate signals. The third column for both tables shows the maximum difference in the energy between the reconstructed and original signals in decibels. In the case of target PSNR $=48 \mathrm{~dB}$ there is only one delay signal that fails to preserve the energy in the last decomposition level (level 9). Fig. 6 shows the energy scaling behavior for the original and the reconstructed signals for all 9 levels of decomposition.

In order to achieve higher PSNR value the EZW algorithm reduces the threshold so that more coefficients in higher decomposition levels are preserved and encoded. If the specified PSNR value can be satisfied without preserving the coefficients in high decomposition levels then the energy in these levels will be significantly distorted. The reason that Table 3 reaches $50 \mathrm{~dB}$ is because that is the target PSNR value for delay signals where the energy is preserved without significant distortion.

The EZW algorithm does not affect the energy of Data rate signals in the same way. This is because data rate signals are much more complex and require preservation of higher frequency components in comparison to the delay signals. For this reason coefficients in higher levels are preserved even in low target PSNR values. The quality however is increased with higher target PSNR values as the threshold decreases and the population of preserved coefficients in all levels of decomposition increases. 


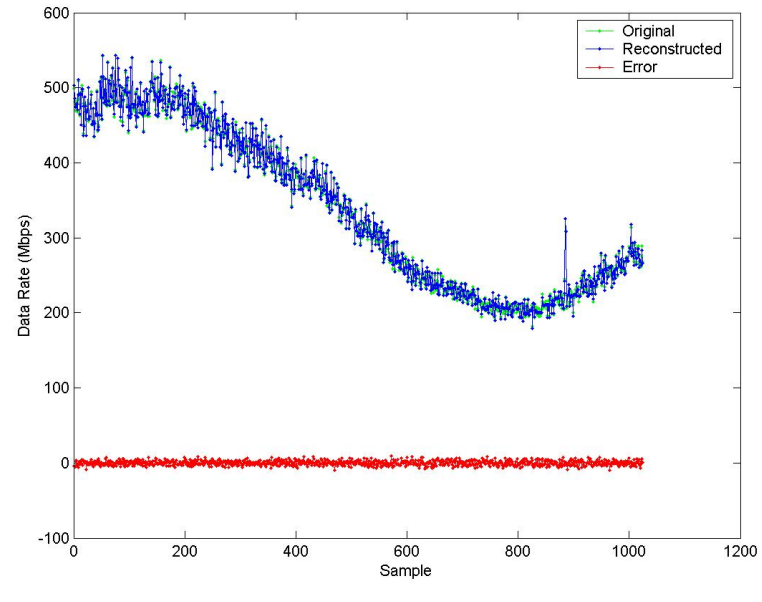

Figure 5. Data rate signal 16 with PSNR $=46.86$ dB and C.R.=12.97

It is interesting to examine the results from the EZW algorithm against a non-transform technique. Bzip2 is considered to be an excellent tool for lossless data compression. Fig. 7 and 8 compare the sizes of the results from the EZW algorithm against the results of bzip2 applied on the original signals. The target PSNR for data rate signals chosen for comparison against bzip2 was $40 \mathrm{~dB}$. This was chosen as it is a balance between the quality metrics and the compression performance. For delay signals the target rate has to be $50 \mathrm{~dB}$ in order to preserve the energy in all scales. On average for delay signals EZW compresses 2.8 times more than bzip 2 and for data rate signals 5.8 times.

\section{Conclusions}

This paper implements the embedded zero-tree algorithm, initially proposed by Shapiro, in order to perform lossy compression on computer network measurements. The algorithm initially encodes the important signal characteristics and then encodes layers of detail until it reaches a user specified quality metric.

EZW can achieve high compression ratios by predicting insignificant wavelet coefficients across different scales while preserving detail in significant signal features and smoothing out the detail information in non-significant parts.

Due to the algorithms feature of controlling the quality of the reconstructed signal, the results are consistent among different signals. The EZW algorithm provides a solution to a problem with statistical based wavelet thresholding algorithms that discarded statistically "hidden" coefficients. Off-line experiments were run using real data rate and de-
Table 3. Average Percentage Relative Error for Standard Deviation, Mean and Maximum Energy difference in Decibel for all $\mathbf{3 0}$ delay signals

\begin{tabular}{|c||c|c|c|}
\hline Target & Std. Err. & Mean Err. & Max. Energy Diff. \\
\hline \hline $50 \mathrm{~dB}$ & $0.07 \%$ & $0.07 \%$ & $0.36 \mathrm{~dB}$ \\
\hline $48 \mathrm{~dB}$ & $0.11 \%$ & $0.13 \%$ & $25.27 \mathrm{~dB}$ \\
\hline $46 \mathrm{~dB}$ & $0.16 \%$ & $0.13 \%$ & $29.2 \mathrm{~dB}$ \\
\hline $44 \mathrm{~dB}$ & $0.25 \%$ & $0.18 \%$ & $29.2 \mathrm{~dB}$ \\
\hline $42 \mathrm{~dB}$ & $0.37 \%$ & $0.27 \%$ & $32.4 \mathrm{~dB}$ \\
\hline $40 \mathrm{~dB}$ & $0.67 \%$ & $0.43 \%$ & $34.1 \mathrm{~dB}$ \\
\hline $38 \mathrm{~dB}$ & $1.04 \%$ & $0.54 \%$ & $34.1 \mathrm{~dB}$ \\
\hline $36 \mathrm{~dB}$ & $1.47 \%$ & $0.67 \%$ & $40.3 \mathrm{~dB}$ \\
\hline
\end{tabular}

Table 4. Average Percentage Relative Error for Standard Deviation, Mean and Maximum Energy difference in Decibel for all $\mathbf{3 0}$ data rate signals

\begin{tabular}{|c||c|c|c|}
\hline Target & Std. Err. & Mean Err. & Max. Energy Diff. \\
\hline \hline $44 \mathrm{~dB}$ & $0.25 \%$ & $0.01 \%$ & $0.15 \mathrm{~dB}$ \\
\hline $42 \mathrm{~dB}$ & $0.24 \%$ & $0.016 \%$ & $0.15 \mathrm{~dB}$ \\
\hline $40 \mathrm{~dB}$ & $0.24 \%$ & $0.016 \%$ & $0.15 \mathrm{~dB}$ \\
\hline $38 \mathrm{~dB}$ & $0.25 \%$ & $0.017 \%$ & $0.15 \mathrm{~dB}$ \\
\hline $36 \mathrm{~dB}$ & $0.17 \%$ & $0.035 \%$ & $0.33 \mathrm{~dB}$ \\
\hline
\end{tabular}

lay measurements.

For an average PSNR value of $42 \mathrm{~dB}$, delay signals are compressed on average 16 times, that is 6 times more than bzip 2 could achieve. For an average PSNR value of 43.7 $\mathrm{dB}$, data rate signals achieve a compression of more than 13 times, that is 5.8 times more than bzip2. Given a specific PSNR value, EZW offers a lower compression ratio for data rate signals, as those signals include much more high frequency components and thus require more detail.

In contrast to data rate signals the EZW algorithm needs to reach much higher PSNR values in order to preserve the energy in all scales. This happens because in the case of delay signals the target PSNR value is reached without needing to include coefficients at higher decomposition levels. In other words the distortion is concentrated in higher levels. On the other hand, for data rate signals due to their bursty nature coefficients need to be preserved from all scales in order to satisfy the target PSNR. So more coefficients in higher levels are preserved and the distortion is 


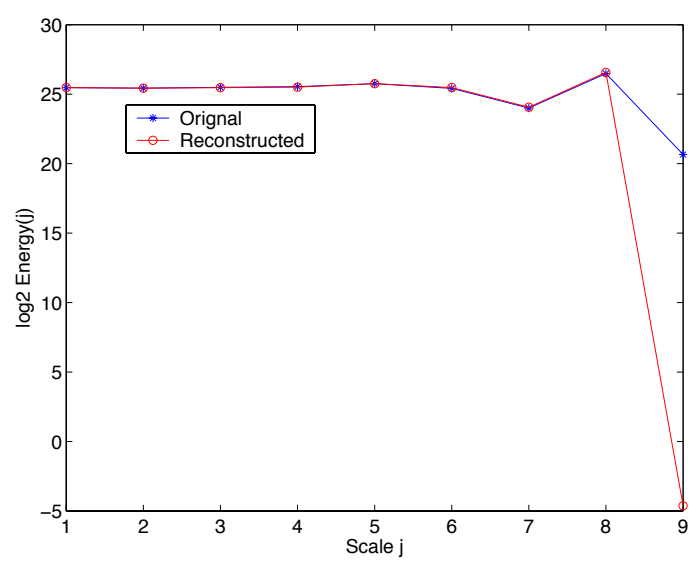

Figure 6. Scaling behavior for delay signal 17 with target PSNR $=48 \mathrm{~dB}$. The difference in the last level is $25.27 \mathrm{~dB}$.

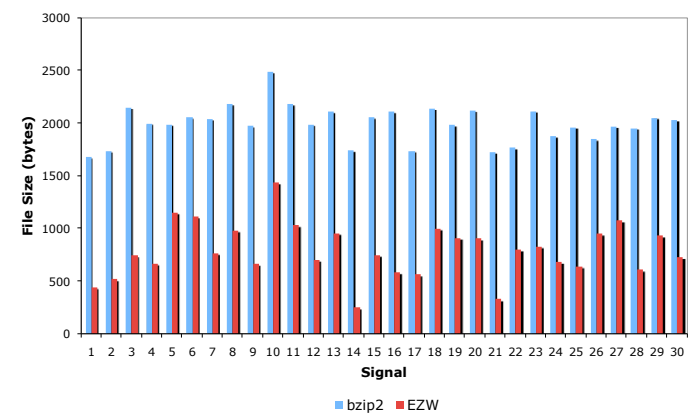

Figure 7. Compression performance of EZT at $50 \mathrm{~dB}$ against bzip2 for delay signals.

spread over all scales.

As for future work, the algorithm could be implemented in a real-time computer network-monitoring tool. A promising candidate seems to be CoMo, a passive monitoring platform developed for the purpose of measuring performance metrics of high speed links and replying to real time queries [7].

A possible additional benefit of the wavelet analysis that is going to be investigated is an anomaly detection scheme for time series processes. Changes in time series processes reveal themselves in various frequency bands in the wavelet domain. By looking at the wavelet coefficients at various scales those changes can be detected.

\section{References}

[1] P. Abry, R. Baraniuk, P. Flandrin, R. Riedi, and D. Veitch. Multiscale nature of network traffic. IEEE Signal Processing Magazine, 19(3):28-46, May 2002.

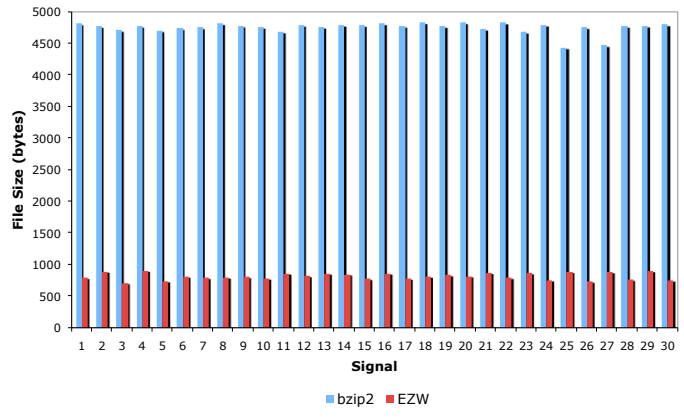

Figure 8. Compression performance of EZT at $40 \mathrm{~dB}$ against bzip2 for data rate signals.

[2] P. Abry and D. Veitch. Wavelet analysis of long-rangedependent traffic. IEEE Transactions on Information Theory, 44(1), January 1998.

[3] J. I. Agbinya. Discrete wavelet transform techniques in speech processing. In Proceedings of the 1996 IEEE Region 10 TENCON - Digital Signal Processing Applications Conference, volume 2, pages 514-519, Perth, Aust, Nov 26-29 1996. IEEE.

[4] T. J. Association. Janet lightpath. Website, 2008. Page visited on 19/12/2008.

[5] P. Barford, J. Kline, D. Plonka, and A. Ron. A signal analysis of network traffic anomalies. In Proceedings of the 2 nd Internet Measurement Workshop (IMW 2002), pages 71-82, 2002.

[6] P. Huang, A. Feldmann, and W. Willinger. A non-intrusive, wavelet-based approach to detecting network performance problems. In Proceedings of the ACM SIGCOMM Internet Measurement Workshop, pages 213-227, November 1-2 2001.

[7] G. Iannaccone, C. Diot, D. McAulley, A. Moore, I. Pratt, and L. Rizzo. The como white paper. Technical report, INTEL, 2004. Page last visited 22/08/05.

[8] M. S. Kim, T. Kim, Y. Shin, S. S. Lam, and E. J. Powers. A wavelet-based approach to detect shared congestion. In ACM SIGCOMM Computer Communication Review, volume 34, pages 293-305, 30 August through 3 September 2004.

[9] K. G. Kyriakopoulos and D. J. Parish. A live system for wavelet compression of high speed computer network measurements. In Proceedings of Passive and Active Measurement (PAM 2007), volume 4427 of Lecture Notes in Computer Science, pages 241-244, Louvain-La-Neuve, Belgium, 4-6 April 2007. Springer-Verlang.

[10] A. Pescappe. Entropy-based reduction of traffic data. Communications Letters, 11(2):3, February 2007.

[11] J. M. Shapiro. Embedded image coding using zerotrees of wavelet coefficients. IEEE Transactions on Signal Processing, 41(1):3445-3462, 1993. 